\title{
Estimation of Brain Activity by Multivariable Auto- regressive Model for Oxy-hemoglobin Changes
}

\author{
Kunihiko OURA*, Kenichi NUMA* and Izumi HANAZAKI** \\ * Department of Health \& Medical Engineering, Faculty of Science and Engineering, \\ Kokushikan University, 4-28-1, Setagaya, Setagaya-ku, Tokyo, 154-8515, Japan \\ **Department of Computers and Systems Engineering, Faculty of Science and Engi- \\ neering, Tokyo Denki University, Ishizaka, Hatoyama-machi, Hiki-gun, Saitama, \\ 350-0394, Japan
}

E-mail:kuni@kokushikan.ac.jp

\begin{abstract}
Estimation of brain activity by multivariable autoregressive model is discussed in the paper. We investigate spontaneous changes in the cerebral oxygenation state by using a multi-channel near-infrared spectroscopy: non-invasive optical topography. Hierarchical decomposition analysis [1] is used to estimate a multivariable autoregressive model. The results are viewed by constructing a brain map that normalizes all the subjects.
\end{abstract}

\section{Introduction}

The purpose of this paper is, to discuss estimation method of brain activity by time-series analysis of data given by a measuring instrument. The instrument used in this paper is near infrared spectroscopy, NIRS. It is well-known for its ability to observe three kinds of hemoglobin densities, $\mathrm{HbO}, \mathrm{HbR}$ and $\mathrm{HbT}$, which reflect blood flow of capillaries in brain.

We have experiments concerned with sense of smell from 20 healthy subjects. Temporal data obtained by experiments show the brain activity of each subject. In addition to some analytical techniques that evaluate transition or correlation of the measurements, we apply estimation method for them and have multivariable autoregressive (MAR) model. The method selected in this paper is hierarchical decomposition analysis, HDA. The results show effectiveness and possibility of using estimated model for oxy-hemoglobin data.

\section{Experiment Setup}

The type of NIRS used for experiments is optical topography system (ETG-7000) manufactured by Hitachi-medico cooperation. There are 15 optical fibers mounted on each probe in $3 \times 5$ array with the distance $30 \mathrm{~mm}$. We evaluate the relative changes in oxy-hemoglobin ( $\mathrm{HbO})$, deoxy-hemoglobin ( $\mathrm{HbR}$ ) and total-hemoglobin (HbT) at sampling period of $100 \mathrm{~ms}$ with the baseline set at the start of the measurement period.

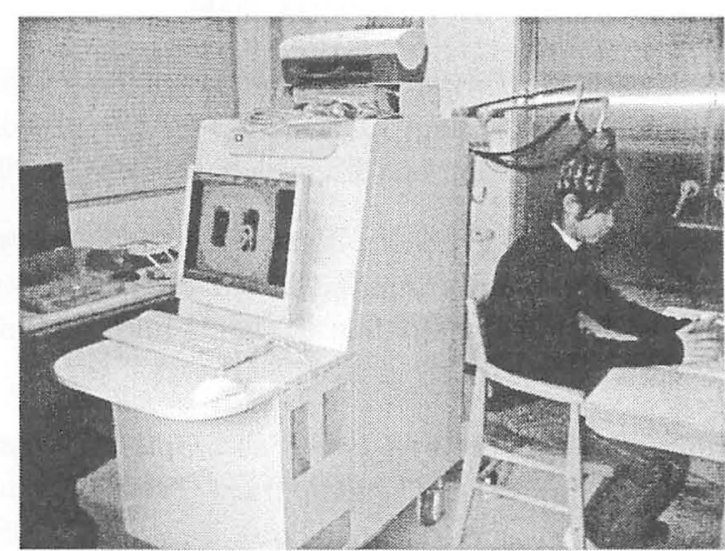

Figure 1. The scene of an experiment.

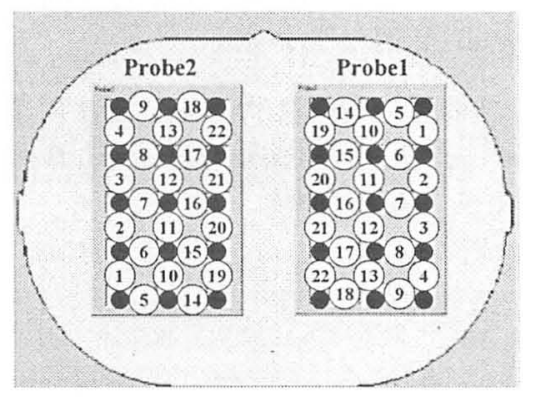

Figure 2. Channels installed on the head

In the experiment, each subject smells three kinds of fragrance in sequence for 60 seconds with his eyes closed. $\mathrm{He}$ does not know the order. One fragrance is a perfume which is said to have "relaxation effect", one is coffee, and the last one is Japanese drink Noni which has pungent odor The experiment adopts the block design shown in figure3, where the subject smells six times so that twice for one fragrance. There are rests between the tasks, each lasts 30 seconds. The data are modified by taking the added mean and moving average of 10 points ( 1 second). Baseline is set as the average of the first rest.

The data are measured from 20 normal subjects (11 men and 9 women, age range 18 to 24 years). All subjects are in good health. 


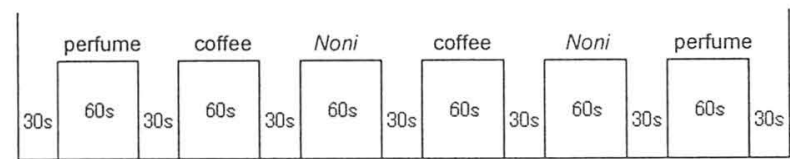

Figure 3. A task schedule for the experiment

\section{Analysis by time-series data}

In this section, we analyze the time-series data by 2 kinds of data analysis technique. Among three kinds of hemoglobin densities, we use only oxidized hemoglobin $\mathrm{HbO}$ for data analysis in the following sections, because it is said to represent brain activity very well.

\subsection{Comparison by average of $\mathrm{HbO}$}

We sum the hemoglobin density of all the channels in each probe and calculate the average of them. It is expected that the difference in volume mean the difference in brain activity. Results are shown in table1, for the first (task.A) and last 30 seconds (task.B) of the task, respectively, where in the first column " $A$ " denotes male and " $B$ " denotes female subject.

We cannot see the difference in three fragrances from table.1, but if we subtract the hemoglobin density of task A from task $B$, we have table2. Table2 shows the difference in two tasks. " 0 " means that the absolute value of the difference is smaller than 0.1 , "- " means smaller than 0.1 and "+" means larger than 0.1 .

We can understand that Noni shows the different property compared with the other fragrances. It is considered that pungent odor strongly affects brain activity, whereas for the other fragrances, brain activity is dependent on subjects' tastes.
Table 2. Difference of $\mathrm{HbO}$ for each task

\begin{tabular}{|c|c|c|c|}
\hline Subject & Perfume & Coffee & Noni \\
\hline A-1 & 0 & 0 & - \\
\hline A-2 & 0 & 0 & - \\
\hline A-3 & + & 0 & + \\
\hline A-4 & 0 & 0 & 0 \\
\hline A-5 & 0 & + & + \\
\hline A-6 & + & 0 & + \\
\hline A-7 & 0 & 0 & 0 \\
\hline A-8 & 0 & + & + \\
\hline A-9 & 0 & 0 & - \\
\hline B-1 & 0 & 0 & - \\
\hline B-2 & + & 0 & - \\
\hline B-3 & 0 & + & 0 \\
\hline B-4 & 0 & - & 0 \\
\hline B-5 & 0 & 0 & + \\
\hline B-6 & 0 & 0 & - \\
\hline B-7 & 0 & 0 & 0 \\
\hline B-8 & + & - & 0 \\
\hline B-9 & 0 & 0 & - \\
\hline B-10 & 0 & 0 & - \\
\hline B-11 & + & 0 & + \\
\hline
\end{tabular}

In order to evaluate the reaction of brain in smaller part, we calculate the average of $\mathrm{HbO}$ for each channel. One of the results is as depicted in figure4, where the left radar graph shows the average in left hemisphere $(22 \mathrm{ch})$ and the right shows the right hemisphere. Most of the results are the same as figure4. that the area of left hemisphere is larger than right hemisphere. It is thought that brain activity of left hemisphere is bigger than that of right hemisphere for the task in this paper. So that we analyze the data only from left hemisphere in this section.

Table 1. Average of $\mathrm{HbO}$ for each task

\begin{tabular}{|c|c|c|c|c|c|c|}
\hline \multirow{2}{*}{ Subject } & \multicolumn{2}{|c|}{ Perfume } & \multicolumn{2}{|c|}{ Coffee } & \multicolumn{2}{|c|}{ Noni } \\
\hline & Task A & Task B & Task A & Task B & Task A & Task B \\
\hline A-1 & 0.137 & 0.123 & 0.284 & 0.318 & 0.892 & 0.438 \\
\hline $\mathrm{A}-2$ & -0.252 & -0.262 & -0.147 & -0.248 & -0.179 & -0.241 \\
\hline A-3 & 0.200 & 0.367 & 0.137 & 0.169 & 0.363 & 0.548 \\
\hline A-4 & 0.315 & 0.359 & 0.299 & 0.266 & 0.225 & 0.262 \\
\hline A-5 & -0.031 & -0.026 & -0.022 & 0.345 & -0.083 & -0.031 \\
\hline A-6 & 0.136 & 0.265 & 0.259 & 0.216 & 0.117 & 0.178 \\
\hline A-7 & -0.123 & -0.082 & -0.101 & -0.115 & -0.140 & -0.180 \\
\hline A-8 & -0.023 & 0.008 & 0.021 & 0.150 & -0.098 & -0.001 \\
\hline A-9 & 0.003 & 0.040 & 0.075 & 0.055 & -0.107 & -0.210 \\
\hline B-1 & 0.004 & 0.028 & -0.044 & -0.037 & 0.039 & -0.100 \\
\hline B-2 & 0.206 & 0.282 & 0.381 & 0.380 & 0.354 & 0.285 \\
\hline B-3 & 0.119 & 0.105 & 0.096 & 0.158 & 0.258 & 0.247 \\
\hline B-4 & 0.030 & -0.015 & 0.056 & 0.001 & 0.076 & 0.069 \\
\hline B-5 & -0.018 & 0.006 & -0.071 & -0.043 & -0.144 & -0.088 \\
\hline B-6 & 0.054 & 0.066 & -0.040 & -0.075 & -0.098 & -0.207 \\
\hline B-7 & 0.078 & 0.070 & 0.078 & 0.073 & -0.005 & -0.019 \\
\hline B-8 & -0.061 & 0.034 & 0.039 & -0.024 & -0.080 & -0.047 \\
\hline B-9 & 0.050 & 0.068 & 0.167 & 0.213 & 0.192 & 0.110 \\
\hline B-10 & 0.194 & 0.206 & 0.297 & 0.266 & 0.483 & 0.353 \\
\hline B-11 & 0.093 & 0.120 & 0.169 & 0.155 & 0.143 & 0.228 \\
\hline
\end{tabular}




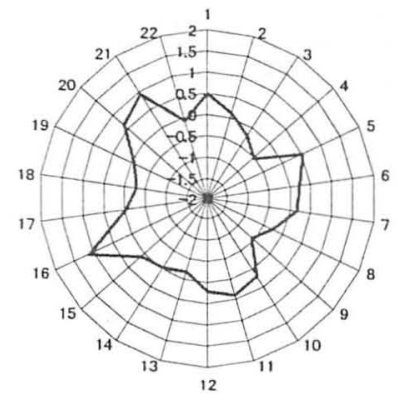

Left hemisphere

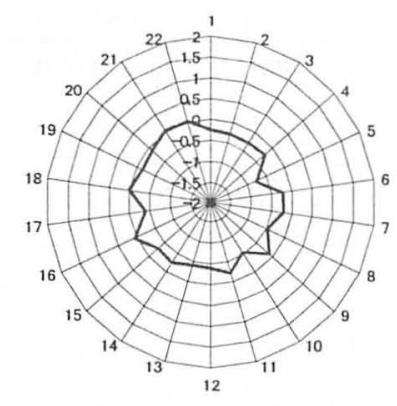

Right hemisphere
Figure 4. Radar graph showing the average of $\mathrm{HbO}$

\subsection{Correlation matrix}

We calculate the correlation coefficients of $\mathrm{HbO}$ between every 2 detector channels for probe in the left hemisphere and thus make a correlation matrix of the data. Calculation of correlation coefficients between $\mathrm{i}$-th and $\mathrm{j}$-th channel are defined by (1).

$$
r_{i, j}=\frac{1}{N} \sum_{i=1}^{N}\left(x_{i}(t)-\bar{x}_{i}\right)\left(x_{j}(t)-\bar{x}_{j}\right)
$$

where $\bar{x}_{i}$ means average of the $\mathrm{i}$-th channel and $N$ is the number of data. Then we have correlation matrix of the left half brain as (2).

$$
R=\left[\begin{array}{cccc}
r_{1,1} & r_{1,2} & \cdots & r_{1,22} \\
r_{2,1} & r_{2,2} & & \vdots \\
\vdots & & \ddots & \vdots \\
r_{22,1} & \cdots & \cdots & r_{22,22}
\end{array}\right]
$$

Correlation matrices for three subjects are shown in figure5 (A-9,B-2,B-10 from left to right and perfume,coffee, Noni from upper to lower). It is mapped as red (warm color, maybe nearly black in gray image) for strong correlation, and mapped as blue (cold color, light in image) for weak correlation.

$$
\text { A-9 }
$$
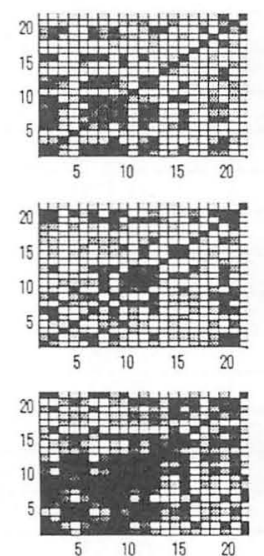

B-2
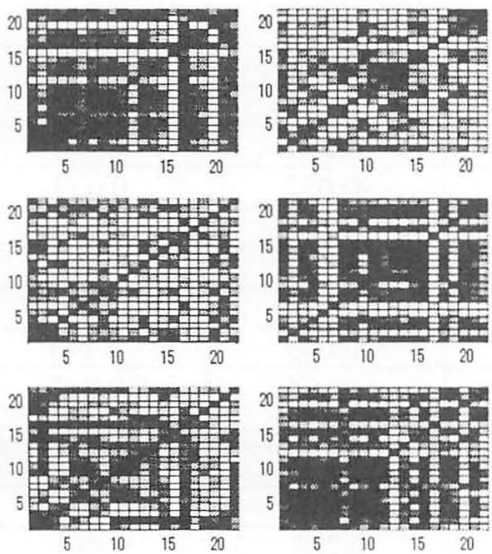

Figure 5. Correlation Matrices
From the figure, it is found that Noni shows the active area around the channel 10 of the left hemisphere, which differs from other fragrances.

\section{Multivariable Autoregression}

Since each subject produces 44 responses ( 22 responses for analysis in this paper) for each experiment, some form of data reduction method is required. We adopt HDA, hierarchical decomposition analysis [1] in this paper.

HDA is one of the data reduction method which resolves the multivariate time-series into hierarchically dependent underlying sources, each driven by noise input and influencing subordinate sources in the hierarchy. It combines principal components analysis, autoregressive modeling, and a novel search strategy among orthogonal rotations. The basic idea in HDA is that the observed data are generated by a set of unobservable basic generators within the brain, so called the HD components.

\subsection{The outline of HD method}

Data vector $X$ is constructed by all the observed $\mathrm{HbO}$ data of the left hemisphere $\left\{x_{i}(n), i=1, \cdots, 22\right\}$ and decompose it by principal components analysis (PCA) as (3) to reduce the umber of components.

$$
X=C^{T} W T
$$

After the reduction of components by suitable level of percent variance, MAR model (4) of the temporal components $T_{p, n}$ is determined by minimizing the sum of squared residual values (5).

$$
\begin{aligned}
& T_{p, n}=R_{p, n}+\mu_{p}+\sum_{q=1}^{P} \sum_{l=1}^{L} A_{q, p, l} T_{q, n-l} \\
& R_{M L A R}=\sum_{p=1}^{P} \sum_{n=1}^{N} R_{p, n}^{2}
\end{aligned}
$$

In (4), $\mu_{p}$ is a mean value, $R_{p, n}$ is a residual value and $A$ is three-dimensional matrix of estimated model parameters given by Yule-Walker equation. $L$ is the model order estimated by Akaike Information Criterion (AIC).

The above mentioned steps give us MAR model of the form (4), however, since the result of PCA decomposition is not unique and may have another model, we must resolve the non-uniqueness problem by following two steps.

The first step is to seek transformation which orthonormalize the innovations $R_{p, n}$. Under the assumptions that $T$ represents underlying generators and each channel of innovations has equal variance, one such matrix can be obtained by dividing the rows of the eigenvectors $R_{p, n} R_{p, n}{ }^{T}$ by the square root of corresponding eigenvalues.

If we denote the transformation matrix as $K$, new temporal components and new estimates are given by the following equations (6),(7). 


$$
\begin{aligned}
& T^{\prime}=K T \\
& A_{\ell}^{\prime T}=K A_{\ell}^{T} K^{-1}
\end{aligned}
$$

The above transformation does not fully resolve the non-uniqueness problem. Then in the next step, we search for a rotation whose transformed temporal components consistent with the hierarchical structure. If $Q$ is denoted as transformation matrix here, $Q$ is obtained iteratively via a procedure similar to Jacobi matrix diagonalization. The algorithm terminates when a full cycle of iterations passes without further reduction of cost function (8).

$$
R_{H D}=\sum_{\ell=1}^{L} \sum_{p=2}^{P} \sum_{q=1}^{p-1}\left[\left(Q A_{\ell}^{\prime} Q^{T}\right)_{p, q}\right]^{2}
$$

Finally, HD components are chosen by using the transformation $Q$ as (9) and (10).

$$
\begin{aligned}
& T_{H D}=Q T^{\prime} \\
& A_{H D}{ }^{T}=Q A_{t}^{\prime} Q^{-1}
\end{aligned}
$$

\subsection{Evaluation of the estimated model}

When we succeed to estimate HD components, upper triangular elements of estimated parameters are weights for feed-forward interactions, the lower triangular elements characterize feed-back interactions. The diagonals are the self-drive weights describing the amount to which a response at one lag contributes to itself. So that it is useful to define the following quantities (11),(12) and (13) in evaluating the estimated model.

$$
\begin{aligned}
& R_{\text {diag }}=\sum_{\ell=1}^{L} \sum_{p=1}^{P} A_{p, p, \ell}^{2} \\
& R_{\text {upper }}=\sum_{\ell=1}^{L} \sum_{p=1}^{P-1} \sum_{q=p+1}^{P} A_{q, p, \ell}^{2} \\
& R_{\text {lower }}=\sum_{\ell=1}^{L} \sum_{p=2}^{P} \sum_{q=1}^{p-1} A_{q, p, \ell}^{2}
\end{aligned}
$$

The numbers of HD components or selected model order often vary each other, which make it difficult to evaluate them as brain activity. One technique to have normalized brain map from HD results concerned with weights. If $T_{H D}$ represents HD components decided from the data vector $X$, we have (14).

$$
X=C^{T} W K^{-1} Q^{-1} T_{H D}
$$

$X$ is observable cerebral $\mathrm{HbO}$ signal and $T_{H D}$ is unobservable basic generators within the brain. Then $C^{T} W K^{-1} Q^{-1}=G$ can be thought to represent a transfer function from basic generators to cerebral oxygenation signal, in a sense, a brain mapping function for the task.

If the number of the subject is $m$, the size of $G$ is $(44 m \times P)$ then normalized function is given by (15), where $H$ is rearrangement of $G$.

$$
\bar{H}(i, j)=\frac{\operatorname{mean}_{k}\{H(i, j, k)\}}{\operatorname{sd}_{k}\{H(i, j, k)\}}
$$

\section{Experiments}

The experimental results are shown in figure6, the original measurements of a male subject, where channel 1 to 7 for upper, 8 to 14 for middle and 15 to 22 to lower.
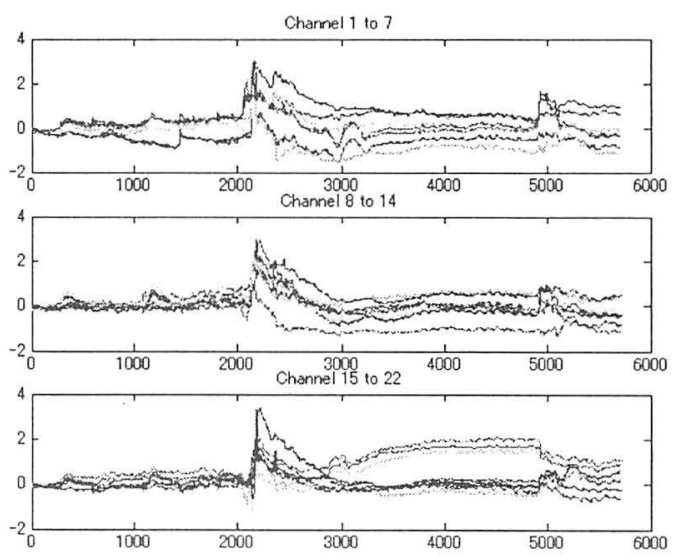

Figure 6. Observed data

By taking the added mean and moving average of 10 points, the data for analysis are given as figure7. where perfume, coffee, Noni from upper to lower.

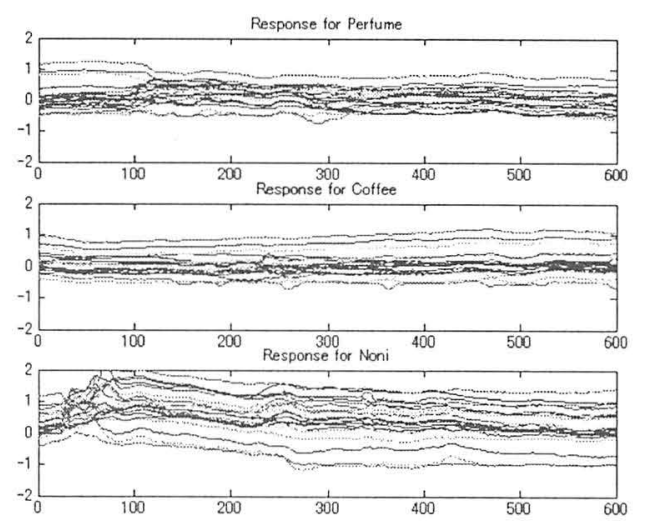

Figure 7. Data after pre-processing

Among three kinds of data, only the result of Noni is applied to HDA in this paper. As described in section4, PCA is applied in the first step. The result of PCA: the percent of variance, ratio of the amount of each component versus the sum of all components, are shown in figure8. We reduce the number of components to 2 from the figure. 


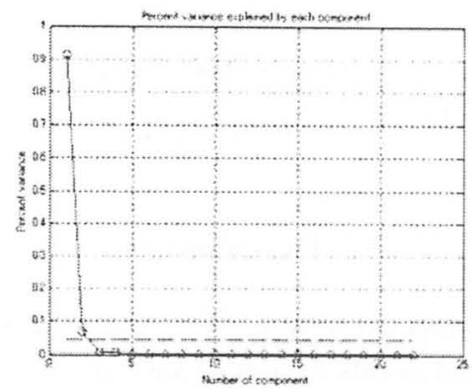

Figure 8. Percent of variance in PCA

HD components calculated by processing the steps in section.5. are shown in figure9.

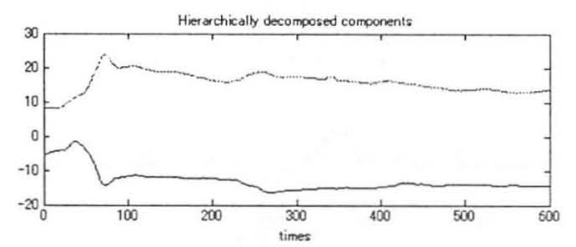

Figure 9. HD components

Original measurements, estimated outputs by HD components and estimated errors are shown in figure.10. Estimates are described by (16) where the order $L=4$. Each estimate is of nearly upper triangular form, which means the hierarchical structure.
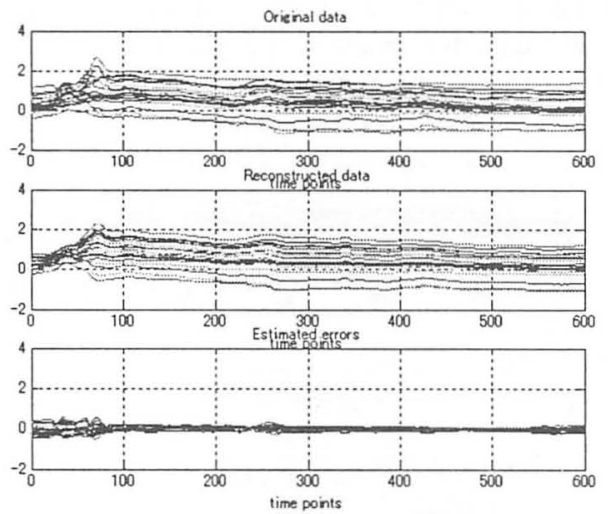

Figure 10. HD components

$$
\begin{aligned}
& A(1)=\left[\begin{array}{cc}
2.119 & 0.172 \\
-0.142 & 2.120
\end{array}\right], A(2)=\left[\begin{array}{cc}
-1.504 & -0.624 \\
0.154 & -1.500
\end{array}\right] \\
& A(3)=\left[\begin{array}{ll}
0.491 & 0.619 \\
0.004 & 0.462
\end{array}\right], A(4)=\left[\begin{array}{cc}
-0.106 & -0.165 \\
-0.017 & -0.084
\end{array}\right]
\end{aligned}
$$

The result is summarized in table 3 . Perfume has more selected components than the others, and the order for coffee is comparatively less than the others.

Table 3. Properties of the estimated model

\begin{tabular}{|c|c|c|c|}
\hline Task & Perfume & Coffee & Noni \\
\hline \hline \multirow{2}{*}{ Components } & $2(50 \%)$ & 2 & 2 \\
& $3(30 \%)$ & & $2(55 \%)$ \\
\hline \multirow{2}{*}{ Order } & $2(50 \%)$ & $2(75 \%)$ & $3(25 \%)$ \\
\hline
\end{tabular}

\section{Conclusion}

The possibility of using multivariable autoregressive model for oxy-hemoglobin data is discussed in the paper. By applying hierarchical decomposition analysis, it is possible to have the typical model of brain activity. Experimental results by using NIRS indicate the difference between tasks, channels and subjects. Normalized brain map shows the active area for each task.

\section{References}

[1] M.A.Repucci, N.D.Schiff and J.D.Victor "General Strategy for Hierarchical Decomposition for Multivariate Time Series: Implications for Temporal Lobe Seizures", Annals of Biomedical Engineering, Vol.29, pp.1135/1149, 2001

[2] L.Kocsis, P.Herman and A.Eke "Mathematical model for the estimation of hemodynamic and oxygenation variables by tissue spectroscopy", Journal of Theoretical Biology, Vol.241, Issue.2, pp.262/275, 2006

[3] K.Oura, T.Iida and K.Numa "Time-series analysis of data measured by Near Infrared Spectroscopy", Proc. of SSS05, pp.78/82, 2005

[4] L.Kocsis, P.Herman and A.Eke "Mathematical model for the estimation of hemodynamic and oxygenation variables by tissue spectroscopy", Journal of Theoretical Biology, Vol.241, Issue.2, pp.262/275, 2006

[5] M.A.Repucci, et.al. "General Strategy for Hierarchical Decomposition for Multivariate Time Series: Implications for Temporal Lobe Seizures", Annals of Biomedical Engineering, Vol.29, pp.1135/1149, 2001 